LA-UR- $09 \cdot 00740$

Approved for public release;

distribution is unlimited.

Title:

Author(s):

Intended for
NLTE Opacities of Mid- and High-Z Cocktails

M.E. Sherrill,

J. Abdallah Jr. ,

H.L. Zhang,

C.J. Fontes,

D.P. Kilcrease.

High Energy Density Physics

Los Alamos National Laboratory, an affirmative action/equal opportunity employer, is operated by the Los Alamos National Security, LLC for the National Nuclear Security Administration of the U.S. Department of Energy under contract DE-AC52-06NA25396. By acceptance of this article, the publisher recognizes that the U.S. Government retains a nonexclusive, royaity-free license to publish or reproduce the published form of this contribution, or to allow others to do so, for U.S. Government purposes. Los Alamos National Laboratory requests that the publisher identify this article as work performed under the auspices of the U.S. Department of Energy. Los Alamos National Laboratory strongly supports academic freedom and a researcher's right to publish; as an institution, however, the Laboratory does not endorse the viewpoint of a publication or guarantee its technical correctness. 


\title{
NLTE Opacities of Mid- and High-Z Cocktails
}

\author{
M. E. Sherrill, J. Abdallah Jr., C. J. Fontes, H. L. Zhang, and D. P. Kilcrease \\ Los Alamos National Laboratory, P.O. Box 1663 Los Alamos, NM 87545
}

\begin{abstract}
In this work we report on the development of a new method for computing mid-and high-Z NLTE opacities. A study has been performed using this method to assess the EOS and opacity sensitivities to the radiation field for both single species $\mathrm{Au}$ and multi-species $\mathrm{SnNb}$ and $\mathrm{U}_{3} \mathrm{Au}$ plasma cocktails with an emphasis on moderately to highly ionized systems. Developed as a benchmark tool to assess both current and future in line NLTE opacity capabilities, we have applied this new approach to assess XSN spectral fidelity for Au at commonly expected NIF hohlraum conditions.
\end{abstract}

Key words:

\section{Introduction}

In the current pursuit of inertial confinement fusion, target designers must contend with a lack of drive energy from current and near future facilities. A considerable effort has been underway to optimize capsulehohlraum performance by pursuing small design improvements. This pursuit for greater performance is the impetus to assess the accuracy of physics packages used in the simulation codes for these systems. This work reports on the development and results from a new midand high- $Z$ computer model intended to eventually become a benchmark code suitable for assessing these approximate methods.

Moderately ionized mid- and high-Z plasmas rely heavily on atomic processes that couple with the radiation field to obtain a state of local thermodynamic equilibrium (LTE). Dielectronic capture followed by spontaneous radiative decay, which is described by a very large rate ${ }^{1}$ is the dommant recombination mechanism driving the system away from LTE. If such a plasma is found to be in a radiation field that has a substantially lower temperature $\left(T_{r}\right)$ than the free electron temperature $\left(T_{e}\right)$, then one will observe a recombined plasma with an ionization balance that is far away from a thermal equilibrium distribution. This shift is clearly seen in Figs. 1 and 2. Fig. 1 illustrates the effects on the ionization distribution for a gold plasma at $\sim 1 / 1000$ solid density. The radiation temperature has been systematically reduced

\footnotetext{
${ }^{1}$ Spontaneous radiative decay rates follows a $Z^{4}$ scaling[1]. Preprint submitted to High Energy Density Physics
}

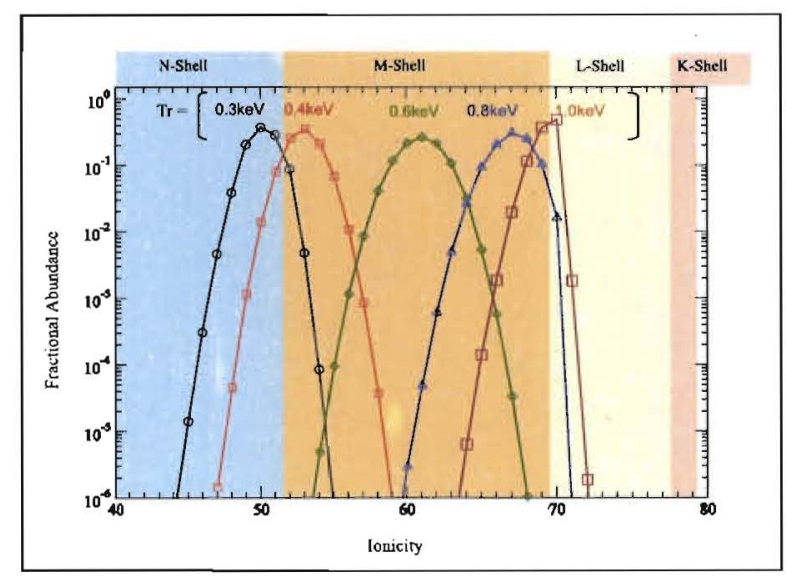

Figure 1: Illustration of the sensitivity of the fractional ionic abundances to several radiation temperatures ranging from $1.0 \mathrm{keV}$ to $0.3 \mathrm{keV}\left(T_{r}\right.$ is reported above each ionization distribution) for a $\mathrm{Au}$ plasma with an atomic number densily $4.748 \times 10^{19} \mathrm{~cm}^{-3}$ and electron temperature $1.0 \mathrm{keV}$.

from the equilibrium value, i.e., $T_{r}=T_{e}=1 \mathrm{keV}$, to a radiation temperature $70 \%$ lower than that of the electron temperature. During this change, the mean ionization has been reduced by more than 20 ion stages. In addition, this figure displays the characteristic broadening in the distribution function as the dominant ion stage moves from a closed shell to the middle of the M-shell with 15 active ionization stages.

A slightly less dramatic case is illustrated at $1 / 10$ February 5, 2009 


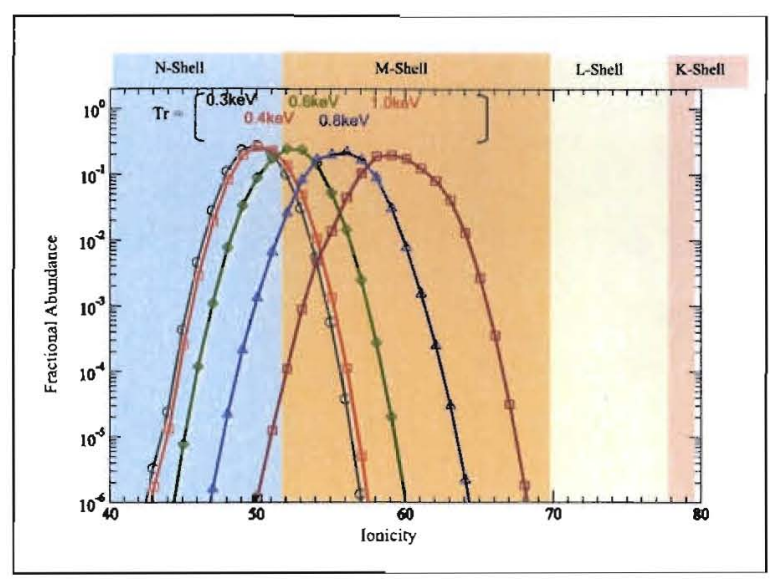

Figure 2: Illustration of the sensitivity of a Au plasma to the fractional ionic abundances for several radiation temperatures ranging from $1.0 \mathrm{keV}$ to $0.3 \mathrm{keV}$ ( $\mathrm{Tr}$ is reported above each ionization distribution) for a plasma with and atomic number density $5.90 \times 10^{22} \mathrm{~cm}^{-3}$ and electron temperature $1.0 \mathrm{keV}$.

solid density in Fig. 2. The lowering of the radiation temperature leads to an 11 ion-stage reduction in the mean ioniciy. In addition, we observe that the ionization balance has lost its sensitivity to the radiation field for $T_{r}<0.4 \mathrm{keV}$, marking the point where the ionization balance is being dominated by the collisional processes.

\section{NLTE opacity modeling}

A method to perform collisional-radiative (CR) modeling has been created and optimized to compute midand high- $Z$ opacities. An Au model has been constructed that includes ions ranging from Rb-like to fully stripped. The atomic structure data contains approximately 250,000 relativistic configurations. Both radiative and collisional processes transfer population between atomic states both within and between adjacent ion stages. Those collisional processes are electronimpact excitation and de-excitation, ionization, and three-body recombination, while the radiative processes include spontaneous radiative decay, stimulated emission and photoionization, radiative recombination, and stimulated recombination. Dielectronic capture and autoionization have also been included. The atomic structure and collisional excitation cross sections and spontaneous radiative decay were computed by Los Alamos National Laboratory's (LANL) RATS code [2] a relativistic Dirac-Fock-Slater code based on the work of
Sampson et al. [3]. Ionizing processes such as photoionization, electron-impact ionization and autoionization were computed by LANL's GIPPER code[2], which is based on the relativistic distorted-wave work of Zhang et al. $[4,5,6]$. All inverse processes were obtained through detailed balance arguments.

This computational method for opacities has two distinct phases of execution: (1) determination of the energy level populations and (2) spectral generation. The construction of a converged high- $Z$ opacity spectrum with relativistic configuration may require tens of millions of configurations. However, one typically finds that only a small minority of these configurations contain non-negligible populations and we will refer to this minority as the equation-of-state (EOS) configurations or states. In regards to the spectral generation phase, these configurations will be the origin of all photoabsorbing transitions ${ }^{2}$.

The vast majority of states, assumed to have no permanent population, will not be included in the kinetic model. They are included as the final transition states (FTS) needed to complete the monochromatic spectrum during the spectral generation phase of the calculation. The construction of the set of FTS begins by selecting a particular EOS configuration. Considering this configuration $^{3}$ as a core configuration, one electron at a time from each occupied core orbital is systematically promoted up to an orbital described by a principal quantum number $n=11$. For each orbital with a vacancy in which the permuted electron may reside, the new configuration is added to the list of FTS. Once all of the core electrons have been permuted from a given EOS configuration, another EOS configuration is selected and the entire process is repeated until the entire list of EOS configurations has been exhausted. Finally, all the duplicate FTS are removed producing a unique set of states.

This approximation of separating states into two classes, i.e., EOS and FTS (See Fig. 3), significantly reduces the size of the rate matrix and amount of collisional and radiative cross section data needed in the kinetic model ${ }^{4}$. However, oscillator strengths and photoionization cross sections are still needed to connect EOS $^{5}$ and FTS configurations in order to generate a

\footnotetext{
${ }^{2}$ Although these states will participate as the final photo-absorbing states in the spectral generation phase, they constitute only a small minority of the total number of final states.

${ }^{3}$ For the first part of this process the core configuration and resulting FTS levels are generated as non-relativistic configurations. Relalivistic configurations are produced during the atomic structure calculations.

${ }^{4}$ With that said, the model is still $120 \mathrm{~GB}$ in size

${ }^{5}$ The term DCA, detailed configuration accounting, and EOS will
} 


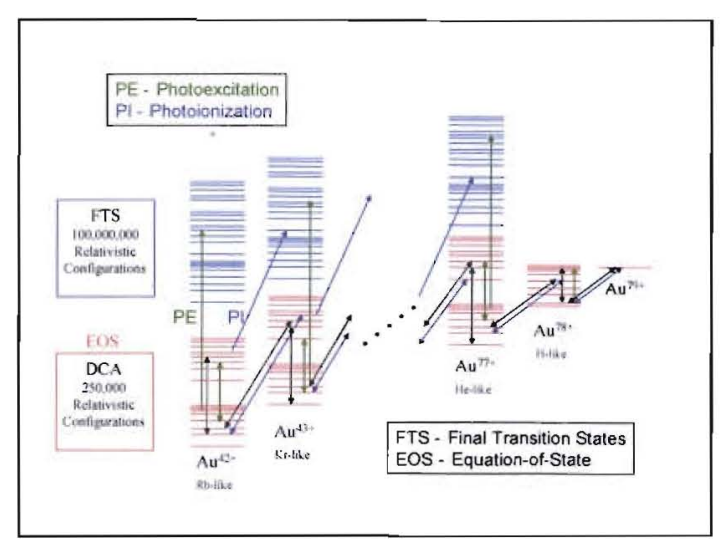

Figure 3: The two-level class structure employed in our model, EOS and FTS, is illustrated in this figure. The EOS states constitute, by definition, configurations containing non-negligible populations and are included in the atomic kinetic rate equations. Photoexcitation and photoionization transitions from EOS to FTS configurations are added during the spectral generation phase of the calculation to complete the opacily spectrum.

complete spectrum.

The effect of adding the final transition states to the opacity spectrum is illustrated in Figs. 4 and 5 . In Fig. 4, above $6 \mathrm{keV}$ the FTS extensions include both the $\mathrm{K}$ and $\mathrm{L}$ bound-free and bound-bound features. Due to computer resource limitations the final configurations of these transitions have been left out the of the set of kinetic rate equations, since they are energetically unfavorable to be included in EOS calculations. However, as clearly seen, they play a significant contribution to the higher photon energy portion of the opacity spectrum. Figure 5 illustrates the filling in of the spectral valleys between photon energies of 100 to $10,000 \mathrm{eV}$ and it is these valleys that have a significant influence on the value of the computed Rosseland mean opacity. These large deficiencies in the opacity spectrum generated with the EOS model are commonly seen at the bound-free edges. Such deficiencies will also be seen later on in this work during the evaluation of the XSN spectra.

\section{Opacity cocktails}

The term cocktail refers to the mixing of atomic elements to produce a custom plasma opacity. Often these mixtures are developed to increase the spectral opacity in a region of interest by selecting two or more elements

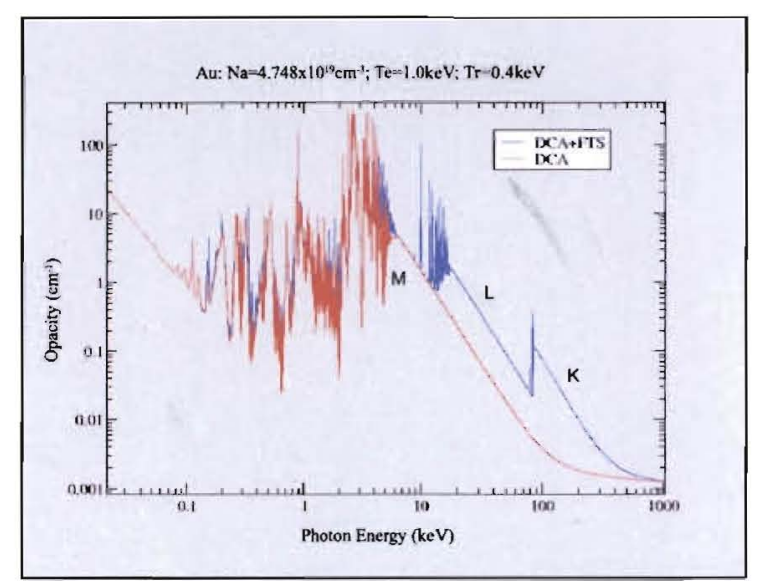

Figure 4: This figure illustrates for a Au plasma the need for the additional FTS configurations. It is apparent for photon energies greater than $2 \mathrm{keV}$ that this large EOS (DCA) model still lacks the capability to produce these structures.

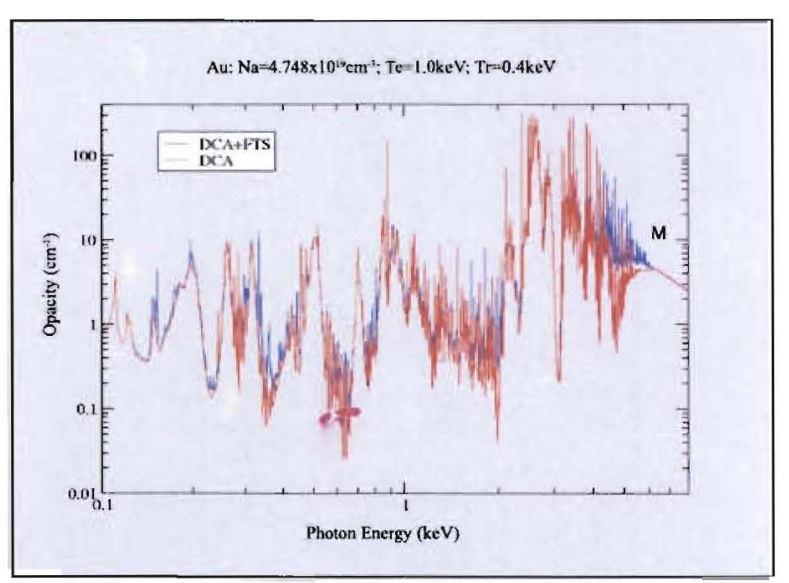

Figure 5: Illustraled here is a narrow spectral region of lower photon energy from the previous figure. The characteristic filling in of the opacity valleys by the addition of the FTS transitions is apparent, especially near the M-shell bound-free edge. 


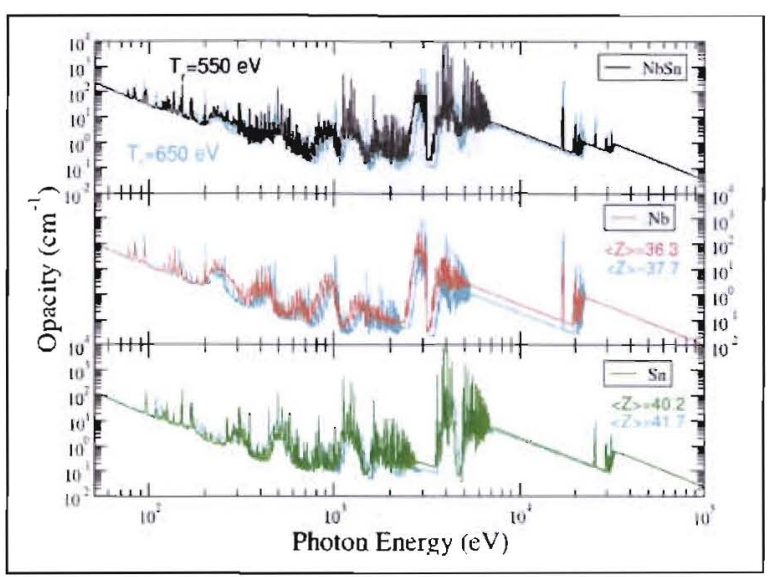

Figure 6: Displayed here in the upper frame are two $\mathrm{SnNb}$ opacity cocktails computed for radiation temperatures of $650 \mathrm{eV}$ and $550 \mathrm{eV}$. The electron temperature $\left(T_{e}=800 \mathrm{eV}\right)$ and atom number density $\left(N_{a}=2.0 \times 10^{20} \mathrm{~cm}^{-3}\right)$ were the same for each. The lower two frames are the spectral contributions of the constituents.

that provide complementary effects where one element would be deficient due to a lack of spectral features.

This point is illustrated in the $\mathrm{SnNb}$ opacity spectrum of Fig. 6. Two total NLTE SnNb opacity spectra are displayed in the upper third of this figure. Each spectrum has been computed with the same atom number density $\left(N_{a}=2.0 \times 10^{20} \mathrm{~cm}^{-3}\right)$ and electron temperature $\left(T_{e}=800 \mathrm{eV}\right)$. The radiation temperatures were chosen to be $550 \mathrm{eV}$ and $650 \mathrm{eV}$. The lower two spectra refer to the individual $\mathrm{Nb}$ and $\mathrm{Sn}$ contributions to the total opacity. In the spectral region centered at $5000 \mathrm{eV}$, a large opacity window is clearly visible for $\mathrm{Sn}$, while non-existent for $\mathrm{Nb}$. The 50:50 binary mixture nearly removes this window. A similar effect is seen at $900 \mathrm{eV}$ and $1700 \mathrm{eV}$. In addition to the complementary nature of the constituent opacities, we see the characteristic shift of spectral features to higher energies as the higher radiation temperatures make more highly ionized ions more plentiful at the expense of the lower ones.

\section{Multi-element atomic kinetics}

The multi-element algorithm used throughout this work follows the development in Ref. [7]. The different species are assumed to coexist in a common pool of free electrons. As described in the algorithm below, the user specifies the atom number density, $N_{a}$, for all $N$ species. Trial values of the total electron density, $N_{\ell}$, are provided for each iteration (i) to the kinetic models of each species. The total system is allowed to iterate until a self-consistent value of $N_{e}$ is obtained. This algorithm is both efficient to execute and simple to implement in a distributed manner due to the relative independence of the kinetic calculations of the individual species. For example, the kinetics of a 15 species plasma has been recently computed with this algorithm [8].

- Specify: $\quad N_{a}^{(1)}, N_{a}^{(2)}, \ldots, N_{a}^{(N)}$ and $\delta N_{e}$

- Initialize: Guess - ${ }^{(0)} N_{e}$

- For $i \geqq 0$ : Solve the set of rate equations to obtain the $\bar{Z}^{(s)}$ for each species,

$$
\begin{gathered}
{ }^{(i)} N_{e} ; k T \rightarrow{ }^{(i)} \bar{Z}^{(1)} \\
{ }^{(i)} N_{e} ; k T \rightarrow{ }^{(i)} \bar{Z}^{(2)} \\
\vdots \\
{ }^{(i)} N_{e} ; k T \rightarrow{ }^{(i)} \bar{Z}^{(N)} \\
{ }^{(i)} N_{a}^{(1)}+{ }^{(i)} \bar{Z}^{(2)} N_{a}^{(2)}+\cdots+{ }^{(i)} \bar{Z}^{(N)} N_{a}^{(N)}={ }^{(i+1)} N_{e}
\end{gathered}
$$

if ${ }^{(i+1)} N_{e} \leqq{ }^{(i)} N_{e}+\delta N_{e}$ then

Multi-element calculation converged! (EXIT For Loop)

else

Increment $i: i=i+1$ (Return to beginning of For Loop)

end if

\section{End For Loop}

This method for computing the energy level populations is based on an ideal plasma assumption that does not take into account non-ideal effects that follow from the reduced space experienced by the plasma ions at higher density.

\subsection{SnNb opacities}

Table 1 illustrates the sensitivity of the Rosseland mean opacity to a systematic decrease in the radiation temperature, while the electron temperature has been held fixed at $800 \mathrm{eV}$. In the most extreme NLTE case, $T_{r}=600 \mathrm{eV}$, the study reveals a $159 \%$ increase in the Rosseland mean opacity. Although the Rosseland weighting function follows the radiation temperature, this effect only accounts for $10 \%$ of this value. In this survey, $\mathrm{Nb}$ shows the largest change in the opacity, though it has the least amount of change in its $\bar{Z}$. This sensitivity in the opacity reflects the large increase 
Table 1: The results presented in this table illustrate SnNb EOS and opacity sensitivity to the radiation temperature. The electron temperature and atom number density were held constant at $800 \mathrm{eV}$ and $2.0 \times 10^{20} \mathrm{~cm}^{-3}$, respectively, while the radiation temperature was systematically reduced. The three percent increases for the Rosseland mean opacity displayed are (1) the total for the cocktail, $\Delta K_{R}^{\mathrm{Tot}},(2)$ the $\mathrm{Nb}$ contribution, $\left(\Delta K_{R}\right)_{N b}$, and (3) the Sn contribution, $\left(\Delta K_{R}\right)_{S n}$.

\begin{tabular}{|c|c|c|c|c|c|c|c|}
\hline & $\tau_{r}(\mathrm{c} \mathrm{V})$ & Gitd. & $\Delta K_{B}^{|l| l(f)}$ & $\left(\Delta K_{R}\right) S_{n}(\%)$ & $\bar{z}_{S n}$ & $\left(\Delta K_{R}\right)_{N b}(\because)$ & $\bar{z}_{N b}$ \\
\hline 1 & 800 & 0.8 & 80 & 0.0 & 44.6 & 000 & 38.6 \\
\hline 2 & 750 & 6.25 & 27.0 & 22.9 & 43.7 & 27.9 & 38.4 \\
\hline 3 & 725 & 9.4 & 42.3 & 35.5 & 43.2 & 46.1 & 383 \\
\hline 4 & 650 & 18 & 48.9 & 60.4 & 41.7 & 131.4 & 37.7 \\
\hline 8 & 600 & 25 & 159 & 98,3 & 40.8 & 233.0 & 37.0 \\
\hline
\end{tabular}

Table 2: The results presented in this table illustrate $U_{3} A u$ EOS and opacity sensitivity to the radiation temperature. The electron temperature and atom number density was held constant at $4000 \mathrm{eV}$ and $5.0 \times 10^{20} \mathrm{~cm}^{-3}$ respectively, while the radiation temperature was systematically reduced. The three percent increases for the Rosseland mean opacity displayed are (1) the total for the cocktail, $\Delta K_{R}^{\text {Tot }}$, (2) the $\mathrm{U}$ contribution, $\left(\Delta K_{R}\right) U$, and (3) the Au contribution, $\left(\Delta K_{R}\right)_{A u}$.

\begin{tabular}{|c|c|c|c|c|c|c|c|}
\hline & $\tau_{r(e V)}$ & Red. & $\Delta K_{R}^{\operatorname{lol}(\xi)}$ & $\left(\Delta K_{R}\right)^{U} U(\%)$ & $\overline{\bar{z}}_{U}$ & $\left(\Delta K_{R}\right)_{A u}\left(\eta_{f}\right)$ & $\overline{\bar{Z}}_{A u}$ \\
\hline$T$ & 4000 & 0.0 & 0.0 & 0.0 & 89.47 & 00 & 76.92 \\
\hline 2 & 3800 & 5.0 & 15.0 & 13.6 & 89.24 & 8.55 & 7690 \\
\hline 3 & 3700 & 7.0 & 24.2 & 22.1 & 89008 & 135 & 76.89 \\
\hline 4 & 3500 & 129 & 47.4 & 430 & 88.65 & 25.4 & 79.84 \\
\hline 5 & 3200 & 200 & 63.2 & 86.6 & 87.66 & 506 & 76.73 \\
\hline
\end{tabular}

in the number of initial bound states that are available for photoabsorption as $\mathrm{Nb}$ recombines from an initially highly ionized He-like LTE ion stage. This behavior is typical in recombining plasmas that are moderately to highly ionized. On the other hand, Sn shows a much larger sensitivity to the drop in the radiation field in regards to $\bar{Z}$. With a mean ionization situated in an open shell, it experiences little resistance to recombining.

\section{2. $U_{3}$ Au opacities}

Currently being considered as cocktail for NIF hohlraums, a simflar study was performed to test the NLTE opacity of $\mathrm{U}_{3} \mathrm{Au}$. The electron temperature of $4000 \mathrm{eV}$ and an atom number density of $5.0 \times 10^{20} \mathrm{~cm}^{-3}$ was chosen to match the conditions where the NIF drive laser interacts with the hohlraum walls. In this case we see a maximum $63 \%$ increase in the Rosseland mean opacity with a $20 \%$ reduction in the radiation temperature in Table 2. Au in this cocktail shows very little change in its $\bar{Z}$ under these plasma conditions, while the U mean charge increases by two ion stages. This overall increase in $\mathrm{U}_{3} \mathrm{Au}$ opacity is good news for ICF designers. Such an increase in opacity leads to fewer energy losses into and through the hohlraum wall[9].

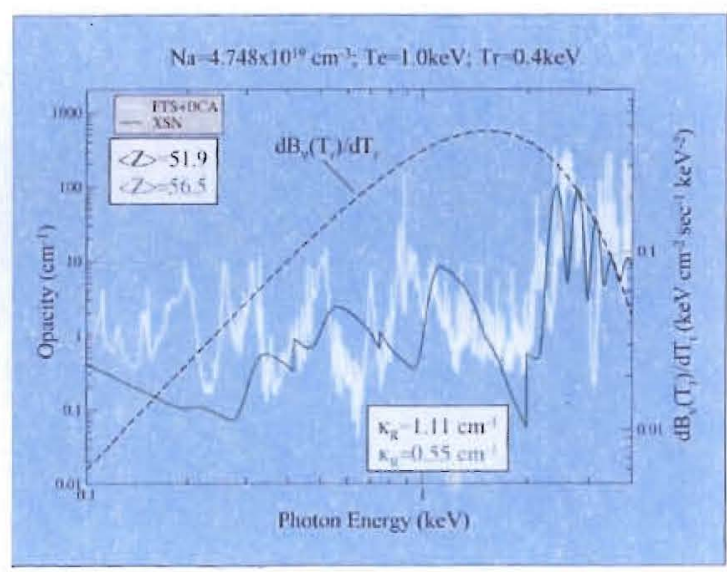

Figure 7: This figure illustrates the difference between the XSN spectral opacity (green) and the detailed calculations (white) for a Au plasma computed at NIF hohlraum conditions $\left(T_{r}=0.4 \mathrm{keV}\right)$. The Rosseland weighting function has been added (dashed line) to emphasize the significant spectral region in the calculation of the Rosseland mean opacity. Apparent is a deep opacity window predicted by XSN that does not exist in the detailed calculations ( $h v=2 \mathrm{keV})$ and the general counter-correlation between these two spectra.

\section{Comparisons with XSN}

XSN [10] is the main NLTE in line opacity package for ICF target designers on both laser driven and pulse power driven facilities in the United States. Though work has been under way to develop alternative packages (see for example Ref. [11]), its short execution time has allowed it to retain its usefulness in particular in the use of 2-D radiation hydrodynamic codes. Figures 7 and 8 compare our detailed NLTE opacity model to XSN for NIF hohlraum conditions of general interest. Both figures were computed with a common atom number density and electron temperature, $4.748 \times 10^{19} \mathrm{~cm}^{-3}$ and $1.0 \mathrm{keV}$, respectively, while the radiation temperature was allowed to vary from $0.4 \mathrm{keV}$ (Fig. 7) to $0.3 \mathrm{keV}$ (Fig. 8). To emphasize the significant region of the opacity spectrum for computing the Rosseland mean opacity, the Rosseland weighting function has been added (see right-hand side axis).

Though in both figures there exist several features in the FTS+DCA spectrum between 0.1 and $0.3 \mathrm{keV}$ that are not presented in the XSN spectrum, these features have little impact on the mean opacity. Ignoring the differences in the prediction of $\bar{Z}$, the most serious spectral discrepancy in regards to the mean opacity exists at the photon energy that corresponds to the maximum of the weighting functions near $2 \mathrm{keV}$. In both XSN spectra, a deep opacity window exists at this photon energy 


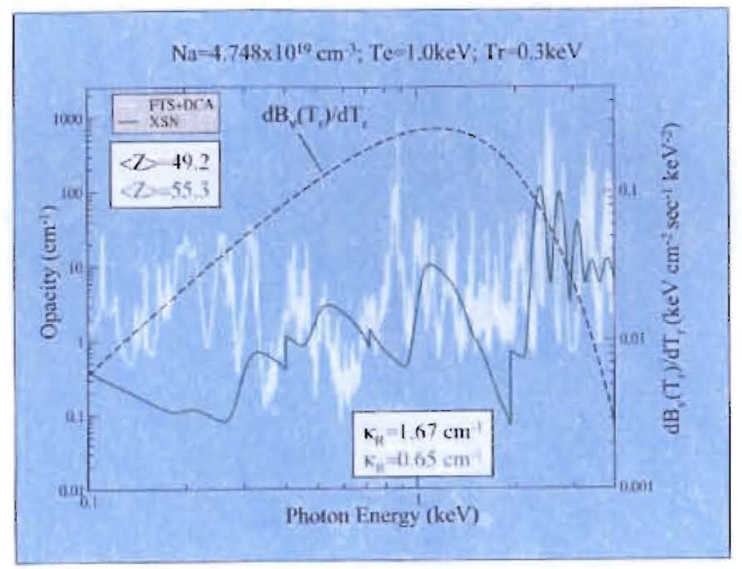

Figure 8: This figure illustrates the difference between the XSN spectral opacity (green) and the detailed calculations (white) for a Au plasma computed at NIF hohlraum conditions $\left(T_{r}=0.3 \mathrm{keV}\right)$. The Rosseland weighting function has been added (dashed line) to emphasize the significant spectral region in the calculation of the Rosseland mean opacity. Apparent is a deep opacity window predicted by XSN that does not exist in the detailed calculations $(h v=2 \mathrm{keV})$ and the general counter-correlation between these two spectra.

which does not exist in the detailed spectra. In addition, one sees a counter-correlation between the detailed and XSN spectra at photon energy points given by 0.3 $0.4 \mathrm{keV}, 0.6 \mathrm{keV}$ and $3.0 \mathrm{keV}$. These discrepancy become more significant when the number of photon energy groups is increased to obtain a more accurate description of the opacity spectrum in order to produce a better converged radiation-hydrodynamic result.

\section{Summary}

In this work we have described the development of a custom NLTE mid- and high-Z opacity method. The purpose of its development is to eventually provide reliable benchmark calculations to assess current and future in-line NLTE opacity models.

In addition, we have provided a quantitative description of the $\bar{Z}$ and opacity sensitivity of mid- and high-Z moderately to highly ionized plasmas, to the radiation temperature. Furthermore, we have provided evidence for the need of a very large number of relativistic configurations inorder to obtain a proper opacity spectrum. Due to restrictions on computational resources, we have developed a two-level class model that reduces the number of levels involved in the kinetics rate model while allowing for the numerous final states needed to properly compute the opacity spectrum. Finally, comparisons between XSN and preliminary results of the model indicate that deep opacity windows predicted by XSN may be unrealistic and may influence detailed radiationhydrodynamic predictions.

\section{Acknowledgments}

We would like to thank Paul Bradley for his discussions on NIF hohlraum conditions. This work was performed under the auspices of the DOE/NNSA Campaign 4 at LANL.

\section{References}

[1] R.D. Cowan, "The theory of atomic structure and spectra", University of California Press.

[2] C.J. Fontes, H.L. Zhang and J. Abdallah, J. J., "An Overview of Relativistic Distorted-Wave Cross Sections", Aromic Processes in Plasmas, Fourteenth APS Topical Conference. Santa Fe, NM 2004, edited by J.S. Cohen, S. Mazevet and D.P. Kilcrease, AIP Conf. Proc. No. 730, (AIP, New York, 2004), p. 41.

[3] D.H. Sampson, H.L. Zhang, A.K. Mohanty and R. E. H. Clark, "A Dirac-Fock-Slater approach to atomic structure for highly charged ions", Phys. Rev. A. 40604 (1.989).

[4] H.L. Zhang and D.H. Sampson, Phys. Rev. A 425378 (1990).

[5] H.L. Zhang, Phys. Rev. A 572640 (1998).

[6] D.H.Sampson and H.L.Zhang, JQSRT, 54345 (1995).

[7] M. E. Sherrill, R. C. Mancini, J. E. Bailey, A. Filuk, B. Clark, P. Lake and J. Abdallah Jr., "Spectroscopic modeling and characterization of a collisionally confined laser-ablated plasma plume". Phys. Rev. E. 76056501 (2007).

[8] J. Colgan, J. Abdallah, Jr. M. E. Sherrill, M. Foster, C. J. Fontes, U. Feldmann, The Astrophysical Journal, 689585 (2008).

[9] O.S. Jones, J. Shein, M. D. Rosen, L. J. Suter, R. J. Wallace, E. L. Dewald S. H. Glenzer, K. M. Campbell, J. Gunther, B. A Hammel, O. L. Landen and C. M. Sorce, R. E. Olson, G. A. Rochau, H. L. Wilkens, J. L. Kaae, J. D. Kilkenny, A. Nikroo, S. P. Regan, "Proof of the principle experiments that demonstrate utility of the cocktail hohlraums for indirect drive ignition", Phys. of Plasmas 14056311 (2007).

[10] W. A. Lokke and W. Grasberger XSNQ-U-A non-LTE Emission and Absorption Coefficient Subroutine, Lawerence Livermore Laboratory Report UCRL-52276 (1977).

[11] J. Abdallah Jr. and M. E. Sherrill. "The reduced detailed configuration accounting (RDCA) model for NLTE plasma calculations". High Energy Density Phys. 4124 (2008) 